\title{
Early Surgery in Femoral Neck Fractures in Elderly: Does Preoperative ASA Score Matter?
}

\author{
Stefania De Sanctis, Raffaella Alonzo, Silvia Frontini, Ilaria Nicolosi, Fabio Belvederi, \\ Edoardo Monaco, Attilio Speranza, Carmelo D'Arrigo
}

Università di Roma La Sapienza, Rome, Italy

Email: stefania.desanctis@hotmail.it

How to cite this paper: De Sanctis, S., Alonzo, R., Frontini, S., Nicolosi, I., Belvederi, F., Monaco, E., Speranza, A. and D’Arrigo, C. (2016) Early Surgery in Femoral Neck Fractures in Elderly: Does Preoperative ASA Score Matter? International Journal of Clinical Medicine, 7, 829-836.

http://dx.doi.org/10.4236/ijcm.2016.712090

Received: October 26, 2016

Accepted: December 24, 2016

Published: December 27, 2016

Copyright $\odot 2016$ by authors and Scientific Research Publishing Inc. This work is licensed under the Creative Commons Attribution International License (CC BY 4.0).

http://creativecommons.org/licenses/by/4.0/

\begin{abstract}
Introduction: Early surgical treatment (within 48 hours) has been recommended for femoral neck fractures in order to avoid complications and reduce mortality rate, regardless of presence and severity of comorbidity and preoperative status (ASA score). However some studies evidenced that early surgery doesn't always have a beneficial effect on mortality and complications. Therefore further studies could be useful in order to better assess risk related factors of patients requiring surgical treatment for femoral neck fracture. The purpose of this study is to evaluate the effect of preoperative ASA score and timing of surgery on mortality, complications and clinical outcome. Methods: All 336 patients operated in our center from January 2013 to December 2014 were selected for this retrospective study. Patients were divided in three groups as follows: group 1 patients treated within 48 hours; group 2 patients treated between 48 to 96 hours; group 3 patients treated over 96 hours. The preoperative ASA score was recorded for each patient. Complication, clinical outcome and mortality at one-year follow-up were evaluated. At follow-up ambulation was graded as: confined to bed, assisted ambulation, and normal ambulation. Complications both local (infections, malunion, dislocation) and systemic (deep vein thrombosis, pulmonary embolism, lung infections, ischemic disorders of heart) were recorded as well as number of transfusions. Statistical analysis was performed with chi square test and $\mathrm{P}$ value $<0.05$ was regarded significant. Results: 308 patients' data were fully available for this study. At one-year follow-up return to normal ambulation was higher for patients of group 1 as compared with group 2 and 3 and in group 2 as compared with group $3(\mathrm{P}=0.04)$. There was no difference in mortality and return to ambulation between patients with ASA score 1 and $2(\mathrm{P}=0.06)$; patients with ASA score $\geq 3$ showed a statistically significant higher mortality $(P=0.004)$ and rate of complications (0.0008) regardless of timing of surgery. There was no statistically significance in blood transfusion among the three groups. Discussion and Conclusion: Clinical outcome, complications and mortality have been previously reported from
\end{abstract}


many authors and most studies agreed that early surgical treatment is recommended regardless of age and preoperative status of the patient. The present study suggests that early surgical treatment is actually able to reduce mortality and complications and to improve clinical outcome in patients with better preoperative conditions, while for patients with ASA score $\geq 3$ treatment within 48 hours seems not to prevent mortality and complications and improve clinical outcome.

\section{Keywords}

Femur Fractures, ASA Score, Early Surgery, Elderly, Hip Surgery

\section{Introduction}

Each year there are 1.5 million femur neck fractures all over the world. They seem to be from 70,000 to 90,000 in Italy each year. Patients involved in these kinds of trauma are mostly elderly patients, with a lot of comorbidity, frailty, difficulties in recovering, and higher risk of surgical complications. For these patients mortality at one year is really high, nearby $12 \%$ - 27\% despite improving in implant technology, surgical techniques, anesthesia, nursing care, and rehabilitation, and mortality during hospitalization seems to be $9 \%$ of the total [1] [2] [3]. About $20 \%$ of patients lose free ambulation capability after femur neck fracture and just $30 \% 40 \%$ of them gain complete recover.

Higher risk of fall in these patients is the reason of higher rate of femur neck fractures; this increased risk may be due to systemic causes such as loss of reflexes, higher risk of lypothymia, pressure changes, circulation alterations, and higher risk of ostheroporosis. All these comorbidities contribute to poor quality of life after femur neck fracture and to reduction of outcome quality. This pathology needs hospitalization and costs of treatment seem to be about 18,000 euros [4].

Last guidelines agree that time to treatment for these fractures is within 48 hours from trauma [5] in order to reduce bedridding. Time to surgery depends on patients' comorbidity and possibility to have operation room, surgical instruments and skilled staff. Other studies evidenced that early surgery has not always a beneficial effect on mortality and complications [3]. Therefore further studies could be useful in order to better assess risk related factors of patients requiring surgical treatment for femoral neck fracture.

There are not in literature studies that evaluate outcome after femur neck fractures in relation to early surgery and patients' ASA score.

Purpose of this study is to evaluate if ASA score influences perioperative mortality and ambulation after femur neck fracture early surgery.

\section{Materials and Methods}

All 336 patients operated in our center from January 2013 to December 2014 were selected for this retrospective study. Mean age of the patients was 83 years, (65 years to 99 years) there were 71 males (21\%) and 265 females (79\%). There were 101 lateral frac- 
tures (30\%) and 235 medial fractures (70\%); 148 right fractures (44\%) and 188 left fractures (56\%). There were 77 arthroprothesis (23\%), 44 hemiarthroplastis (13\%), 20 screws (6\%), 7 hip plates (2\%) and 188 femur neck nails (56\%). The mean length of stay was 11 days. Patients were divided in three groups as follows: group 1 patients treated within 48 hours, group 2 patients treated between 48 to 96 hours, group 3 patients treated over 96 hours. The preoperative ASA score was recorded for each patient. Complication, clinical outcome and mortality at one-year follow up were evaluated. At follow up ambulation was graded as: confined to bed, assisted ambulation, normal ambulation. Complications both local (infections, malunion, dislocation) and systemic (deep vein thrombosis, pulmonary embolism, lung infections, ischemic disorders of heart) were recorded as well as number of transfusions. Statistical analysis was performed with chi square test and $\mathrm{P}$ values $<0.05$ was regarded significant. This study was approved by an ethic commette and each patient signed the consense. There were no missing patients at the follow up or incomplete datas.

\section{Results}

308 patients data were fully available for this study. Data are showed in Table 1.

Table 1. Results divided in three groups and ASA score.

\begin{tabular}{|c|c|c|c|c|}
\hline & & ASA 1 & ASA 2 & ASA 3 \\
\hline \multirow[t]{5}{*}{$<48 \mathrm{H}$} & Mortality & $0 \%$ & $5 \%$ & $45 \%$ \\
\hline & Bed confined & $0 \%$ & $5 \%$ & $11 \%$ \\
\hline & Assited ambulation & $0 \%$ & $25 \%$ & $22 \%$ \\
\hline & Normal ambulation & $100 \%$ & $13 \%$ & $22 \%$ \\
\hline & Complications & $0 \%$ & $70 \%$ & $22.20 \%$ \\
\hline \multirow[t]{5}{*}{$48 \mathrm{H}-96 \mathrm{H}$} & Mortality & $25 \%$ & $17 \%$ & $50 \%$ \\
\hline & Bed confined & $0 \%$ & $3 \%$ & $4 \%$ \\
\hline & Assited ambulation & $25 \%$ & $26 \%$ & $23 \%$ \\
\hline & Normal ambulation & $50 \%$ & $54 \%$ & $23 \%$ \\
\hline & Complications & $25 \%$ & $60 \%$ & $29 \%$ \\
\hline \multirow[t]{11}{*}{$>48 \mathrm{H}$} & Mortality & $40 \%$ & $32 \%$ & $52.00 \%$ \\
\hline & Bed confined & $0 \%$ & $7 \%$ & $4 \%$ \\
\hline & Assited ambulation & $40 \%$ & $22 \%$ & $24 \%$ \\
\hline & Normal ambulation & $20 \%$ & $39 \%$ & $20 \%$ \\
\hline & Complications & $27 \%$ & $50 \%$ & $50 \%$ \\
\hline & total & & & \\
\hline & Mortality & $41 \%$ & $31 \%$ & $\star_{50 \%}$ \\
\hline & Bed confined & $15 \%$ & $18 \%$ & $53 \%$ \\
\hline & Assited ambulation & $35 \%$ & $30 \%$ & $25 \%$ \\
\hline & Normal ambulation & ${ }^{\star} 45 \%$ & $52 \%$ & $22 \%$ \\
\hline & Complications & $5 \%$ & $9 \%$ & ${ }^{\star} 20 \%$ \\
\hline
\end{tabular}

*Statistical significance. 
Mortality increases of $15 \%$ from group A to B and of $10 \%$ from group B to C. The amount of bedridden patients and with poor deambulation (wheelchair, medical walker) is constant among the three groups.

The amount of ambulanting patients (free ambulation, crutches) reduces by $10 \%$ from each group to the other. The difference is statistically significance for deambulation ( $P: 0.0398$ ) even if for mortality is nearby significance (P: 0.0599). Analyzing groups divided by ASA, in the group with ASA 1 the percentage of bedridden patients doesn't vary; percentage of ambulating patients increases of $25 \%$ from group A to group B and another 20\% from Group B to Group C. Ambulating patients reduce of $50 \%$ from Group A to Group B and of 30\% from Group B to Group C. Days of hospital stay increase proportionally to time before surgery. These differences are not statistically significance.

Analyzing ASA 2 group the mortality patients increases of $10 \%$ from Group A to Group B and from Group B to Group C.

Bedridden and not ambulating patients number remains constant among the three groups. Ambulating patients number reduces of 10\% from Group A to Group B and $15 \%$ from Group B to Group C.

Days of hospital stay increase proportionally to time to surgery. These differences are not statistically significance even if for mortality is nearby significance (P: 0.0599).

Analyzing ASA 3 group mortality increases of 5\% from Group A to Group B, it doesn't vary from Group B to Group C.

Bedridden patients percentage decrease of 5\% from Group A to Group B and it doesn't vary from Group B to Group C.

Ambulating patients number doesn't vary among the three groups.

Days of hospital stay increases according to time to surgery.

These differences have not statistically significance.

Analyzing groups only on ASA score, mortality percentage decreases of $5 \%$ from ASA 1 to ASA2 and increase of $30 \%$ from ASA 2 to ASA 3. The number of bedridden patients increases of 5\% from ASA 1 to ASA 2 and ASA 3.

Chi square test shows significance for mortality (P: 0.0040) and ambulation (P: 0.0008).

Complications percentage in group A lesser than groups B and C, that have the same complications percentage one each other. This difference have not statistically significance.

In ASA 1 group complications number increases of 25\% from Group A to Group B and of $15 \%$ from Group B to Group C.

These differences haven't statistically significance.

In ASA 2 group complications increase from Group A to Group B of $10 \%$ and of $10 \%$ from Group B to Group C. These differences haven't statistically significance.

In ASA 3 group complications number decreases of $10 \%$ from Group A to Group B and decreases of $15 \%$ from Group B to Group C.

These differences haven't statistically significance. 
Analyzing complications only in ASA score groups they increase od 10\% from ASA 1 to ASA 2 and 20\% from ASA 2 to ASA 3.

These differences have statistically significance.

Group A presents lower percentage of transfusion, lesser than $10 \%$ then the other groups. These differences have not statistically significance.

ASA 1 group needs 20\% less transfusion of the other groups. Chi Square test isn't significance.

Blood Transfusions number increases from Group A to Group B of 25\%, it decreases of $10 \%$ from Group B to Group C. Chi square test is not significant.

In ASA 3 group percentage of blood transfusions is over $50 \%$. Chi Square test is not significance.

ASA 2 and ASA 3 patients present increase of $30 \%$ of blood transfusion then ASA 1 Group. Chi square is a bit over significance (P: 0.0733).

\section{Discussion}

In last years, some studies tried to find correlation between time to surgery and patients outcome, but findings are not univocal.

Some studies report improvement of complications and mortality at one year:

In the retrospective study of Moja et al. [1] 35 different articles were compared showing a reduction of mortality and pressure lesions in patients treated within 48 hours.

In a retrospective study of Khan' et al. [3] over three days of wait, there was an increase of mortality, morbidity and hospitalization. In this study seems that waiting for improving hydration causes an increase of pressure lesions, thrombo-embolisms and infections.

Uzoigwe et al. [2] presented a study comparing post-operative results of patients treated at 12-24-36-60-72 hours from hospitalization; results reported increasing of mortality during hospitalization and at 1 year in delayed surgery, and found an increase of pressure lesion risk proportional to patients ASA score.

Our study is in agreement with these works, reporting increase of mortality of $10 \%$ among groups A and B and of complications in ASA 1 and ASA 2 groups whit delayed surgery, but not for ASA 3 group where delayed surgery seems to reduce complications.

Other works found increase of mortality with delayed surgery but not a relation with complications:

Todd et al. [6] found a significance increase of mortality but not of morbidity in patients treated after $48 \mathrm{~h}$.

Dorotka et al. [7] compared surgery at 6-12-18-24-36 hours and recommended surgery within 36 hours after which there was an increase of mortality.

This work has same results for mortality that increases with delayed surgery and transfusions after surgery, but does not agree on complications.

Some authors found better results in early surgery only on complications but not on mortality. 
Parker et al. [8] in their study found an increase of pressure lesions, pulmonary embolism, lung infections and hospitalization associated with increase of time before surgery. They also found a decrease of mortality in patients treated with arthroplasties and hemiarthroplasties; they attributed this difference on early mobilization of patients treated with prosthesis.

A German study of Smektala et al. [9], showed an increase of complications but not of mortality in patients treated after 36 hours. Complications observed were pressure lesions, urinary infections, thromboembolism, cardiovascular accidents, post-operative bleeding and implant complications.

Pillay et al. [10] showed a reduction of pulmonary infections in patients with delayed treatment but not of the mortality that seemed to be influenced only by preoperative conditions of the patients.

Our study showed an increasing of mortality with delayed surgery but only for patients ASA1 and ASA2.

Choi et al. [11] did not find significant differences of mortality and post-operative complications among groups treated within or after 3 days from hospitalization.

Our work does not agree with these conclusions but for post-operative transfusion.

Our results shows that death patients' percentage increases of $15 \%$ from Group A to Group B and other 10\% from Group B to Group C. There seems not to be differences between bedridden patients, patients on wheelchair and patients with medical walker among three groups. There are differences in ambulating patients, with or without crutches: increase of $10 \%$ of mortality from each group to the other with increasing of time to surgery.

Looking at data significance, it was possible demonstrate by Chi Square test a significant difference for ambulation (P: 0.0398) and nearby significance for mortality ( $P$ : 0.0599). This may be due to small number of patients, however $P$ value obtained is nearby significance and let suppose a potential correlation between these events analyzed.

In order to analyze the influence of clinical conditions on clinical outcome, data were organized on ASA score classification. The study of Yeho and Fazal analyzed the effect of ASA score on time to surgery and patients outcomes, were time to surgery was secondary to patients conditions and operating theater availability [12]. Our results agrees with the results of this study in therms of hospital stay and complications, but in our study is also evaluated the effect of early surgery on patients outcomes, even in patients with ASA 3 or more.

Analyzing data for patients ASA 1 mortality increases of $25 \%$ from Group A to Group B and other 20\% from Group B to Group C; contemporary there is a massive reduction of ambulation capability with increasing of time to surgery.

Evaluating ASA 2 patients variations of mortality and ambulation ability has the same trend of ASA 1 patients, with $10 \%$ of difference among three groups.

ASA 3 patients have 8\% increase of mortality between Group A and Group B.

Comparing ASA score without division in groups based on time to surgery, mortality 
and ambulation show a decrease: of $10 \%$ of mortality from ASA 1 to ASA 2 and an increase of $30 \%$ from ASA 2 to ASA 3; ambulation does not change from ASA 1 to ASA 2 and decrease of $30 \%$ from ASA 2 to ASA 3.

These suggests a role of patients clinical status on final outcome, and a predominant influence of time to surgery on ASA 1 patients outcome; a middle influence in ASA 2 and a marginal effect on ASA3, where survivor is already affected by a strong presence of comorbidity.

Looking at postoperative complications, Group A showed a percentage lesser of 5\% then Group B and C that has the same percentage of post-operative complications.

Evaluating data on ASA score:

In ASA 1 and ASA 2 patients with the increasing of time to surgery, there is an increasing of post-operative complications; $15 \%-20 \%$ among ASA 1 Groups and 10\% ASA 2.

In ASA 3 patients with the increasing of time to surgery complications reduces of $20 \%$ from Group A to Group C.

This suggests that also for post-operative complications, benefits of early surgery are more evident for ASA 1 and ASA2 patients, and that a short time to surgery for ASA 3 increases early complications.

Looking at complications on ASA score, removing Groups based on time to surgery, there is an increase of $10 \%$ from ASA 1 to ASA 2 and of 20\% from ASA 2 to ASA 3. These differences are statistically significance at Chi Square Test.

These data show a strong influence of pre-operative clinical conditions of patients on post-operative early complications that matches with influence of early surgery.

Looking at blood transfusions: in Group A, blood transfusions are 10\% less than other groups.

Evaluating patients on ASA classification we can see that in ASA 1, patients that need blood transfusion are $20 \%$ less than the total.

In ASA 2 group percentage increases from Group A to Group B of $25 \%$ and decreases of $10 \%$ from Group B to Group C.

In ASA 3 patients there is not influence of time to surgery, and transfusion need is just above $50 \%$.

Comparing data only on ASA score, ASA 2 and ASA 3 patients show an increase of $30 \%$ of blood transfusion then ASA 1 patients, Chi Square is just above significance.

This suggests a higher influence of pre-operative clinical conditions of the patients on blood transfusion need, and a non-relevant influence of time to surgery.

\section{Conclusions}

Results of the study in patients with femur neck fractures show an increase of mortality and a worst ambulation capability associated with increasing of time to surgery in patients with ASA score 1 and 2. ASA score has shown to have influence on mortality and ambulation capability.

Early complications are shortened by early surgery in ASA 1 and ASA 2 groups, not 
in ASA 3 where they increase. Even complications are influenced by pre-operative conditions, more than mortality and ambulation.

Blood transfusions are not influenced by early surgery, but seem to be influenced by ASA score; there is an increase of blood transfusions with increasing of ASA score.

The short number of patients and retrospectivity represent limit of this study, even if significant results suggest that with a higher number of patients results will be stronger.

This study suggests that early surgery is a good choice for patients with ASA score 1 and 2, but for patients with ASA 3 is better evaluate the option of delayed surgery.

Other studies will be useful to find a correct guideline to better treat these patients.

\section{References}

[1] Moja, L., Piatti, A. and Banfi, G. (2012) Timing Matters in Hip Fracture Surgery: Patients Operated within 48 Hours Have Better Outcomes Meta-Analysis and Meta-Regression of over 190.000 Patients. PLOS ONE, 7, e46175. https://doi.org/10.1371/journal.pone.0046175

[2] Uzoigwe, C., Burnand, H. and Cheesman, C. (2013) Early and Ultra-Early Surgery in Hip Fracture Patients Improves Survival. Injury, 44, 726-729.

https://doi.org/10.1016/j.injury.2012.08.025

[3] Khan, C., Kalra, S. and Khanna, A. (2009) Timing of Surgery for Hip Fractures: A Systematic Review of 52 Published Studies Involving 291.413 Patients. Injury, 40, 692-697. https://doi.org/10.1016/j.injury.2009.01.010

[4] Prasar, A. (2011) Fracture in the Enderly: When Is Hip Replacement Necessary? Clinical Interventions in Aging, 6, 1-7.

[5] Mark, J.C., Cameron, I.D. and March, L.M. (2010) Evidence-Based Guidelines for the Management of Hip Fractures in Older Person: An Update. Medical Journal of Australia, 192, 37-41.

[6] Todd, C.J., Palmer, C., Camilleri-Ferrante, C., Freeman, C.J., Laxton, C.E., Parker, M.J., Payne, B.V. and Rushton, N. (1995) Differences in Mortality after Fracture of Hip. BMJ, 311, 1025. https://doi.org/10.1136/bmj.311.7011.1025b

[7] Dorotka, R., Schoechtner, H. and Buchinger, W. (2003) The Influence of Immediate Surgical Treatment of Proximal Femoral Fractures on Mortality and Quality of Life. Journal of Bone and Joint Surgery, 85, 1107-1113. https://doi.org/10.1302/0301-620X.85B8.14282

[8] Parker, M. and Pryor, G. (1992) The Timing of Surgery for Proximal Femoral Fractures. Journal of Bone and Joint Surgery, 74, 203-205.

[9] Smektala, R. and Endres, H. (2008) The Effect of Time-to-Surgery on Outcome in Elderly Patients with Proximal Femoral Fracture. BMC Musculoskeletal Disorders, 9, 171. https://doi.org/10.1186/1471-2474-9-171

[10] Pillay, J., van der Wouden, J.C. and Leenen, L.P. (2007) Retrospective Application of the Performance Indicator Hip Fracture: Operated within 24 Hours in 217 Patients Treated at the University Medical Center Utrecht in 2000-2003: Reduction in Postoperative Pneumonia but Not Mortality. Nederlands Tijdschrift voor Geneeskunde, 151, 967-970. https://doi.org/10.4103/0019-5413.144232

[11] Choi, H. and Kim, E. (2014) The Timing of Surgery and Mortality in Elderly Hip Fracture. Indian Journal of Orthopaedics, 48, 599-604. https://doi.org/10.1177/2151458514560471

[12] Yeoh, C.J.C. and Fazal, M.A. (2014) ASA Grade and Elderly Patients with Femoral Neck Fracture. Geriatric Orthopaedic Surgery \& Rehabilitation, 5, 195-199. 
Submit or recommend next manuscript to SCIRP and we will provide best service for you:

Accepting pre-submission inquiries through Email, Facebook, LinkedIn, Twitter, etc. A wide selection of journals (inclusive of 9 subjects, more than 200 journals)

Providing 24-hour high-quality service

User-friendly online submission system

Fair and swift peer-review system

Efficient typesetting and proofreading procedure

Display of the result of downloads and visits, as well as the number of cited articles

Maximum dissemination of your research work

Submit your manuscript at: http://papersubmission.scirp.org/

Or contactijcm@scirp.org 